\title{
Hypoxia enhances the production and antitumor effect of exosomes derived from natural killer cells
}

\author{
Yanan Jiang ${ }^{1,2 \#}$, Haiming Jiang ${ }^{3 \#}$, Kun Wang ${ }^{1}$, Chunling Liu ${ }^{2}$, Xuejing Man ${ }^{4}$, Qiang Fu ${ }^{1,5,6}$ \\ ${ }^{1}$ School of Pharmacy, Binzhou Medical University, Yantai, China; ${ }^{2}$ Department of Immunology, Binzhou Medical University, Yantai, China; ${ }^{3}$ Intensive \\ Care Unit, Yantai Affiliated Hospital of Binzhou Medical University, Yantai, China; ${ }^{4}$ Department of Ophthalmology, Yantai Yuhuangding Hospital, \\ Yantai, China; ${ }^{5}$ School of Medicine, University of North Carolina at Chapel Hill, North Carolina, USA; ${ }^{6}$ Shandong Cellogene Pharamaceutics Co. \\ LTD, Yantai, China \\ Contributions: (I) Conception and design: Q Fu, X Man; (II) Administrative support: Q Fu, X Man, H Jiang; (III) Provision of study materials: Y Jiang, \\ H Jiang; (IV) Collection and assembly of data: Y Jiang, K Wang; (V) Data analysis and interpretation: C Liu; (VI) Manuscript writing: All authors; \\ (VII) Final approval of manuscript: All authors. \\ \#These authors contributed equally to this work. \\ Correspondence to: Qiang Fu. No. 346 Guanhai Road, Laishan District, Yantai 264003, China. Email: qiangfu11@fudan.edu.cn; Xuejing Man. \\ Department of Ophthalmology, Yantai Yuhuangding Hospital, Yantai, China. Email: manxuejing@aliyun.com.
}

Background: Exosomes are a subgroup of extracellular vesicles that are naturally released by almost all types of cells. However, the factors that promote the capacity of natural killer (NK) cells to release exosomes are unclear. In this study, we investigated whether hypoxia can enhance the yield of NK cell-derived exosomes and improve the immunotherapeutic effects of these cells.

Methods: Exosomes from NK92 or NK92-hIL-15 cells were isolated from culture medium under normoxic (NK92-Exo and NK92-hIL-15-Exo) or hypoxic (hypoxic NK92-Exo and hypoxic NK92-hIL-15Exo) conditions. NK92-Exo and hypoxic NK92-Exo were characterized by transmission electron microscopy (TEM), nanoparticle-tracking analysis (NTA), and western blot. Real-time cell assay, wound healing assay, flow cytometry, and western blot were then performed to assess cytotoxicity, cell proliferation, cell migration, apoptosis, and the expression levels of cytotoxicity-associated proteins.

Results: After 48 hours of hypoxic treatment, NK92-Exo exhibited significantly increased cytotoxicity, enhanced inhibition of cell proliferation, and elevated levels of molecules associated with NK cell cytotoxicity. The hypoxia-treated NK92-Exo and NK92-hIL-15-Exo showed increased expression of three functional proteins of NK cells—specifically FasL, perforin, and granzyme B-as compared with their NK92-Exo counterparts exposed to normoxia.

Conclusions: As an approach that supports overproduction of exosomes, hypoxic treatment of NK cells may serve as a promising therapeutic option for cancer immunotherapy.

Keywords: Hypoxia; extracellular vesicles; exosomes; natural killer cells; cancer therapy

Submitted Dec 28, 2020. Accepted for publication Mar 02, 2021.

doi: $10.21037 / \mathrm{atm}-21-347$

View this article at: http://dx.doi.org/10.21037/atm-21-347

\section{Introduction}

Natural killer (NK) cells are regarded as the body's first line of defense against viral and bacterial infection, and can exert cytotoxic effects without prior antigen sensitization (1). According to the levels of CD56 and CD16 expression on their surface, NK cells can be divided into two subsets:
CD $56^{\text {dim }}$ CD $16^{\text {bright }} \mathrm{NK}$ cells $(80-95 \%$ of NK cells) and CD $56^{\text {bright }}$ CD $16^{\text {dim }} \mathrm{NK}$ cells (10-15\% of NK cells) (2-4). $\mathrm{CD}^{\text {dim }}{ }^{\text {CD } 16^{\text {bright }}}$ NK cells display high levels of CD16 expression and often play a role in antibody-dependent cellmediated cytotoxicity (ADCC), while $\mathrm{CD} 56^{\text {bright }} \mathrm{CD} 16^{\text {dim }}$ NK cells play a vital role in immunomodulation through 
their secretion of numerous immune regulatory factors including interferon-gamma (IFN) $-\gamma$ and tumor necrosis factor-alpha (TNF- $\alpha)$ (5). Upon recognition of their target cells, NK cells may directly or indirectly play a role in cytotoxicity. One such mechanism of NK cells involves the activation of apoptotic pathways through Fas-Fas-ligand interaction involving TNF-associated death ligands or activated receptors (6). Another pro-apoptotic mechanism involves the release of cytotoxic vesicles containing proapoptotic enzymes such as granzyme B and perforin (7). In our study, NK92 cells, which belong to the CD56 bright NK subset, were used, because their clinical application is supported by their safety profile and approval by the United States Food and Drug Administration (FDA) (8-10).

Extracellular vesicles (EVs), including exosomes, are derived naturally from live cells under certain physiological and pathological conditions (11). These extracellular vesicles exist in serum, cerebrospinal fluid, breast milk, and other body fluids, and are broadly classified into three major groups on the basis of their size and function: exosomes (30-150 nm), microvesicles (MVs, 150-1,000 nm), and apoptotic bodies (1,000-5,000 nm) (12). Exosomes contain an abundance of genetic materials and proteins, and are able to regulate the transmission of information. They have attracted considerable attention due to their involvement in a variety of diseases as well as their potential for use as promising diagnostic and therapeutic tools (13). Exosomes from NK cells (NK-Exo) have been reported to contain NK-specific proteins and have antitumor effects, as demonstrated in both in vitro and in vivo models $(14,15)$. However, the factors responsible for promoting the release of exosomes from NK cells remain unclear.

Hypoxia is a key feature shared by multiple types of solid tumors, and it contributes to tumor development, drug resistance, and distant metastasis $(16,17)$. In the tumor microenvironment, following initial exposure of tumor cells to hypoxic conditions, numerous signaling pathways may be activated to enable tumor cell survival and adaptation. However, persistent hypoxia results in cell death $(17,18)$. One response to hypoxia that permits cellular adaptation is the release of hypoxia-inducible factors (HIFs), which are a family of transcription factors $(19,20)$. Interestingly, Patton et al. observed that hypoxia in pancreatic tumors promoted the release and uptake of exosomes, which contributed to enhanced cancer cell survival under hypoxic conditions (21). Moreover, in NK cells, the expression of activated receptors on the surface is decreased under hypoxic conditions, as is cytotoxicity $(22,23)$. However, the effects of hypoxia on the biogenesis of exosomes from NK cells have not been reported, and it is unclear whether these exosomes differ from those derived from normoxic NK cells. In the present study, we established models of human cancers to investigate whether hypoxia can enhance the yield of NK cell-derived exosomes and improve the immunotherapeutic effect.

We present the following article in accordance with the MDAR reporting checklist (available at http://dx.doi. org/10.21037/atm-21-347).

\section{Methods}

\section{Cell culture}

MCF-7 (human breast cancer) and A2780 (human ovarian cancer) cell lines supplied by the American Type Culture Collection (ATCC, Manassas, VA) were cultured in highglucose Dulbecco's Modified Eagle's Medium (DMEM, HyClone, Logan, UT, USA) with 10\% fetal bovine serum (Gibco, Grand Island, NY, USA) and 1\% penicillinstreptomycin (Hyclone, Logan, UT, USA) added. The human NK cell line NK92, which was isolated from a 50-year-old man with malignant non-Hodgkin lymphoma and successfully established by Klingemann's group in 1992 (24), was also obtained from the ATCC. The NK92 cell line was cultured in serum-free medium for lymphocyte (Dakewei, Beijing, China) supplemented with $20 \%$ fetal bovine serum, $200 \mathrm{IU} / \mathrm{mL}$ recombinant human interleukin (IL)-2, and $1 \%$ penicillin-streptomycin. Human IL-15 genemodified NK92 cells (NK92-hIL15) were obtained from our laboratory and incubated in serum-free medium for lymphocyte supplemented with $10 \%$ fetal bovine serum and $1 \%$ penicillin-streptomycin. All cells were cultured at $37^{\circ} \mathrm{C}$ and cultures were performed under either normoxic or hypoxic conditions. To create hypoxic conditions, cells were cultured in a hypoxic workstation incubator (Thermo Fisher Scientific, Waltham, MA, USA), incorporating a gas mixing system containing a mixture of $1 \% \mathrm{O}_{2}, 5 \% \mathrm{CO}_{2}$ and $94 \% \mathrm{~N}_{2}$.

\section{Exosome isolation}

NK92 cells were cultured in serum-free medium for 48 hours under hypoxic or normoxic conditions. To isolate pure exosomes, the supernatants were collected, and cells and debris were removed through centrifugation at increasing speeds as follows: $300 \times \mathrm{g}$ for 10 minutes, $2,000 \times \mathrm{g}$ for 10 minutes, and $10,000 \times g$ for 30 minutes at $4{ }^{\circ} \mathrm{C}$. The supernatant was then passed through a $0.22-\mu \mathrm{m}$ filter 
before centrifugation at $100,000 \times \mathrm{g}$ for 70 minutes to pellet the exosomes using clear ultracentrifuge tubes (Beckman, CA, USA) (25). The exosome pellets were washed twice in a large volume of phosphate-buffered saline (PBS) and then recovered by centrifugation at $100,000 \times \mathrm{g}$ for 1 hour, after which the exosomes were resuspended in PBS for the experiments to follow. The protein content of the normoxic NK92-exosomes (NK92-Exo), the hypoxic NK92-exosomes (hypoxic NK92-Exo), and the hypoxic NK92-hIL-15 exosomes (hypoxic NK92-hIL-15-Exo) was determined using a bicinchoninic acid protein assay kit (Solarbio, Beijing, China).

\section{Exosome characterization}

The morphological characteristics of purified exosomes were verified by transmission electron microscopy (TEM) using a JEM-1400 TEM (JEOL, Tokyo, Japan) operated at $100 \mathrm{kV}$. The sizes of NK92-Exo and hypoxic NK92Exo were determined using a NanoSight NS300 (Malvern Instruments, Malvern, UK).

\section{Western blot analysis}

Whole-cell lysates or exosomal proteins were separated using sodium dodecyl sulfate-polyacrylamide gel electrophoresis and transferred onto polyvinylidene difluoride membranes. After being blocked with 5\% non-fat milk in tris-buffered saline with Tween at room temperature for 2 hours, the blots were incubated overnight at $4{ }^{\circ} \mathrm{C}$. Following treatment with the corresponding primary antibodies at dilutions recommended by the suppliers, the blots were incubated with horseradish peroxidaseconjugated secondary antibodies (Abbkine, Redlands, CA, USA) at room temperature for 1.5 hours. The blots on the membranes were developed using enhanced chemiluminescent detection reagents. CD63 and TSG101 (ab134045, ab125011; Abcam, Cambridge, UK,) were used as exosome markers. Antibodies against HIF-1 $\alpha$ (ab51608; Abcam, Cambridge, UK), FasL (\#68405, CST, MA, USA), perforin (ab97305; Abcam, Cambridge, UK), and granzyme B (ab134933; Abcam, Cambridge, UK) were used as functional markers of NK cells. GAPDH (Proteintech, Wuhan, China) was used as a loading control.

\section{Real-time unlabeled cell analysis technique}

MCF-7 and A2780 cell proliferation was analyzed with real-time cell analysis (RTCA). MCF-7 and A2780 cells, used as targeted cells, were observed every 5 minutes after incubation using the xCELLigence system E-Plate. Cell index (CI) values were recorded and proliferation was observed continuously for 120 hours. Cell proliferation capacity was determined by calculating the difference in CI compared to the controls and analyzing the slope of the curve between 0 and 120 hours (26).

\section{Wound healing assay}

The migration ability of targeted cells (MCF-7 and A2780 cells) was measured by performing wound healing assay. Four groups of cells were included in the experiment: (I) PBS; (II) NK92-Exo; (III) 24-hour hypoxic NK92-Exo; and (IV) 48-hour hypoxic NK92-Exo. Photos were then taken under an inverted microscope, the initial width of the scratch was measured, and the culture was continued at $37{ }^{\circ} \mathrm{C}$ in a $5 \% \mathrm{CO}_{2}$ incubator. After 48 hours, photos were again taken at the same observation point, the scratch width was measured, and cell mobility was calculated using ImageJ software (NIH, USA). Mobility $=$ (initial scratch width current scratch width)/initial scratch width $/ 2 \times 100 \%$. The treatment protocol described above was used for both the A2780 cells and the MCF-7 cells.

\section{Analysis of apoptosis by flow cytometry}

To verify the cytotoxic effects of NK92-Exo and hypoxic NK92-Exo on MCF-7 and A2780 cells, Annexin V-allophycocyanin (APC)/7-aminoactinomycin D (7-AAD) double staining was performed using an Annexin V-APC Kit (Biolegend, CA, USA). MCF-7 and A2780 cells were preincubated with PBS, NK-92 Exo, 24-hour hypoxic NK92-Exo, and 48-hour hypoxic NK92-Exo for 24 hours, harvested, and washed. The samples were then centrifuged, the supernatants discarded, and the cells resuspended in binding buffer. The stains were added and gently mixed with the cells, which were subsequently incubated at room temperature in the dark for 15 minutes. Binding buffer $(400 \mu \mathrm{L})$ was added to each tube before analysis by flow cytometry (BD Biosciences).

\section{Statistical analysis}

SPSS17.0 statistical software (Chicago, USA) was used to analyze and process the obtained data. Measurement data from 3 separate experiments were represented by 

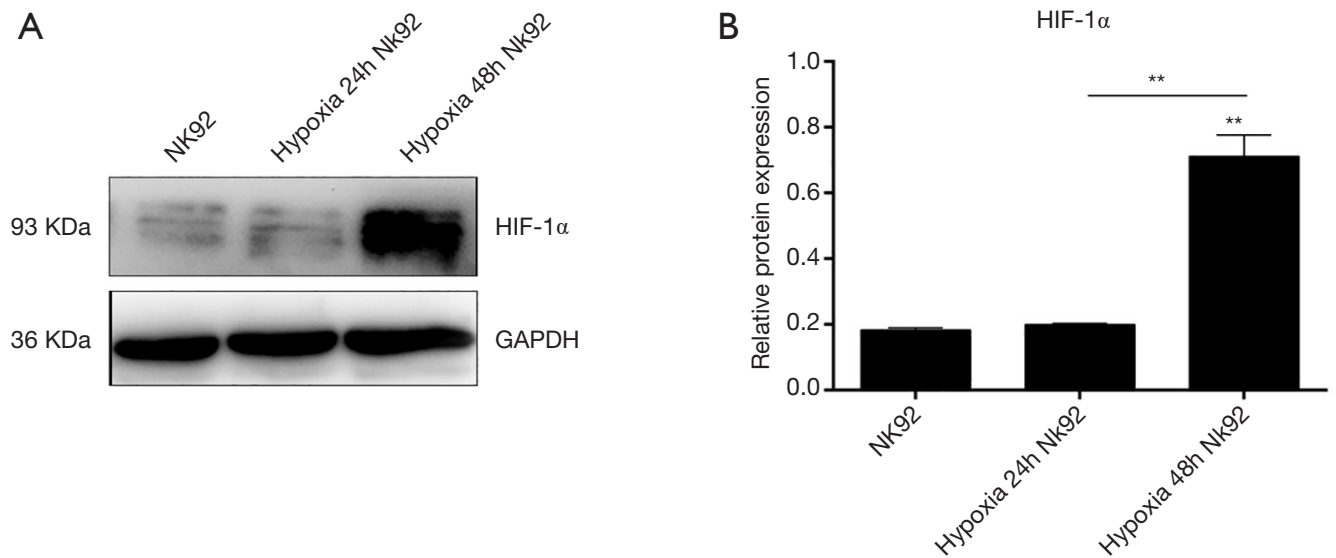

Figure 1 Expression of HIF-1 $\alpha$ on hypoxia NK92 cells. Western blotting was used to evaluate HIF-1 $\alpha$ expression in NK92 cells under normoxic $\left(20 \% \mathrm{O}_{2}\right)$ and hypoxic $\left(1.0 \% \mathrm{O}_{2}\right)$ conditions. Western blotting analysis of HIF-1 $\alpha$ expression in NK92, 24-hour hypoxic NK92, and 48-hour hypoxic NK92 cells (30 $\mu \mathrm{g}$ of total protein were used for each cell group) (A). Relative protein expression of HIF-1 $\alpha$ in hypoxic NK92 cells compared to GAPDH (B). Results were obtained from 3 separate experiments, and the means \pm SDs were plotted. ** $\mathrm{P}<0.01$.

means \pm standard deviations. Comparisons between two groups were statistically analyzed by $t$-test, and multiplegroup comparisons were analyzed by analysis of variance (ANOVA). A $\mathrm{P}$ value $<0.05$ was considered to represent statistical significance.

\section{Results}

\section{Expression of HIF-1a in bypoxic NK92 cells}

We first cultured NK92 cells under normoxic conditions, or under hypoxic conditions for 24 or 48 hours, and then detected HIF-1 $\alpha$ expression in hypoxic NK92 cells (Figure 1A). The expression of HIF-1 $\alpha$ in NK92 cells increased with the duration of hypoxic treatment, reaching its highest level after 48 hours $(\mathrm{P}<0.01$, Figure $1 B)$.

\section{Characterization of NK92-Exo in hypoxic and normoxic conditions}

TEM analysis revealed that all exosomes consisted of spherical particles enclosed within a teacup-like membranous structure, which was in accordance with the recognized characteristics of exosomes (Figure $2 A$ ). As observed through nanoparticle-tracking analysis (NTA), the diameter of NK92-Exo, 24-hour hypoxic NK92-Exo, and 48-hour hypoxic NK92-Exo was 205.6 $\pm 29.65,196.8 \pm 17.37$, and $239.2 \pm 10.03 \mathrm{~nm}$, respectively (Figure $2 B$ ). These results demonstrate that hypoxia did not obviously change the morphology or size of the exosomes. Western blot was carried out to further analyze the protein expressions of NK92-Exo and hypoxic NK92-Exo. The results revealed the presence of the exosomal marker proteins CD63 and TSG101, whereas the control protein GAPDH was hardly expressed, and these findings were consistent with those of previous studies (Figure 2C,D). Overall, these results indicated that NK92-Exo and hypoxic NK92-Exo had been successfully isolated from the culture medium.

\section{Hypoxia enhances the release of exosomes from NK92 cells}

To assess exosome production under different conditions, NK92 cells were incubated under either normoxic $\left(20 \% \mathrm{O}_{2}\right)$ conditions for 48 hours or hypoxic $\left(1.0 \% \mathrm{O}_{2}\right)$ conditions for 24 or 48 hours, after which the culture medium was collected. Our results indicated that from the $10^{8}$ NK92 cells maintained under normoxic conditions for 48 hours, $0.78 \pm 0.058 \mu \mathrm{g}$ of exosomes was obtained. After 24 and 48 hours of hypoxic culture, the same number of NK92 cells had produced $1.00 \pm 0.059$ and $1.96 \pm 0.298 \mu \mathrm{g}$ of exosomes, respectively. The amount of protein particles increased more than 2-fold in exosomes from hypoxia-treated cells. As compared with those obtained under normoxic conditions, the levels of exosomes in NK92 cells were increased after $24(\mathrm{P}<0.05)$ and $48(\mathrm{P}<0.01)$ hours of hypoxic treatment (Figure $2 E$ ). Furthermore, a significantly greater number of exosomes was produced in the 48 -hour hypoxic treatment 
A

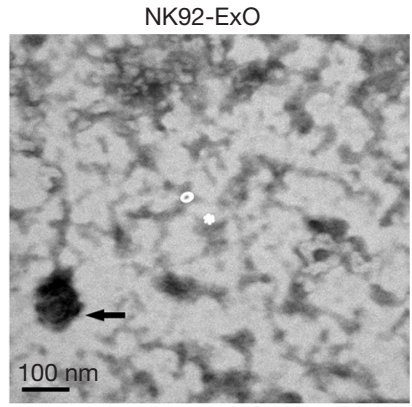

B

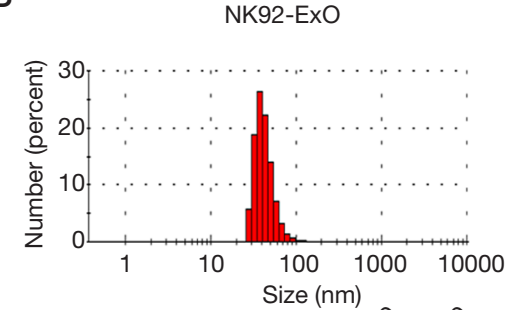

C

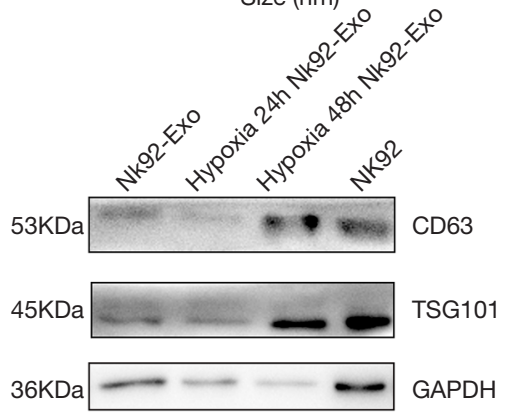

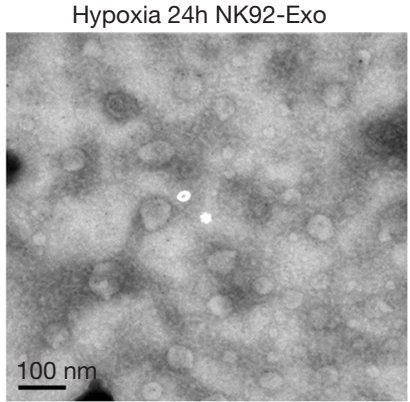

Hypoxia 24h NK92-Exo

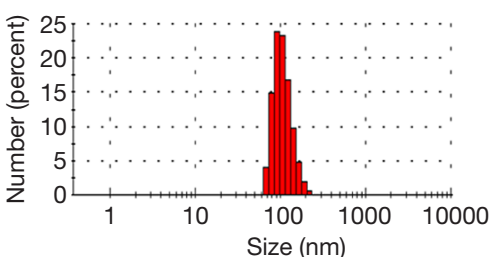

$\mathrm{D}$

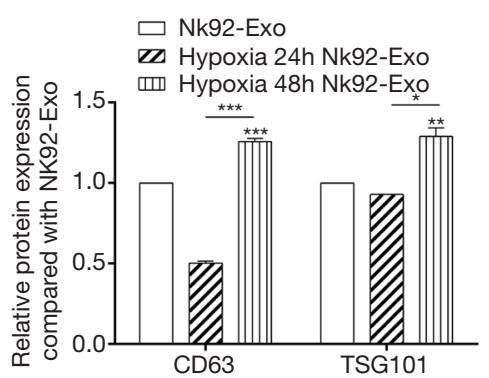

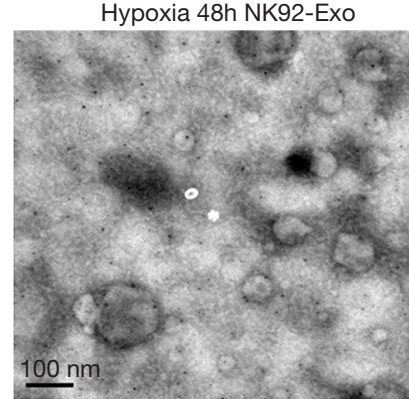

Hypoxia 48h NK92-Exo

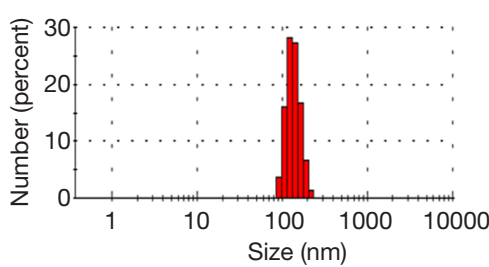

$\mathrm{E}$

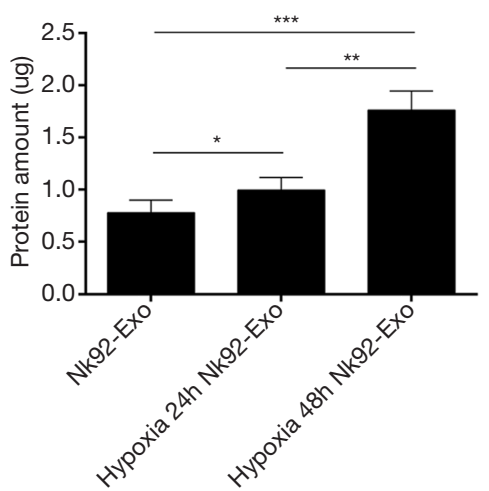

Figure 2 Characterization of NK92-Exo and hypoxic NK92-Exo. Characteristics of exosomes from cell supernatants under normoxic (20\% $\left.\mathrm{O}_{2}\right)$ and hypoxic $\left(1.0 \% \mathrm{O}_{2}\right)$ conditions were determined by nanoparticle tracking analysis, transmission electron microscopy, and western blot. Transmission electron microscopy was used to evaluate the phenotype of exosomes (scale bar, $100 \mathrm{~nm}$ ) (A). Nanoparticle tracking analysis imaging of NK92-Exo and hypoxic NK92-Exo indicated that the size distributions of NK92-Exo and hypoxic NK92-Exo were approximately $200 \mathrm{~nm}$ (B). The expression of the exosomal marker proteins CD63 and TSG101 was confirmed by western blot analysis in NK92-Exo and hypoxic NK92-Exo (30 $\mu$ g of total protein was used for both cells and exosomes) (C). Relative protein expression compared with NK92-Exo of CD63, and TSG101 in NK92-Exo and hypoxic NK92-Exo (D). Yields of NK92-Exo and hypoxic NK92-Exo were determined as the total amount of protein obtained from $10^{8} \mathrm{NK} 92$ cells $(\mathrm{E})$. Results were obtained from 3 separate experiments, and the means \pm SDs were plotted. *, $\mathrm{P}<0.05$; **, $\mathrm{P}<0.01$; **, $\mathrm{P}<0.001$.

group than in the 24-hour hypoxic treatment group $(\mathrm{P}<0.05)$.

\section{Enhancement of in vitro cytotoxic effects on cancer cells under hypoxia NK92-Exo}

The cytotoxic effects of NK92-Exo and hypoxic NK92-
Exo on MCF-7 breast cancer and A2780 ovarian cancer cells were evaluated by RTCA. Hypoxic NK92-Exo had a time and dose-dependent cytotoxic effect on MCF-7 and A2780 cells, with the cell index value of the targeted cells decreasing as the concentration and duration of incubation increased (Figure $3 A, B, C, D$ ). With the concentration of exosomes at 25 or $50 \mu \mathrm{g} / \mathrm{mL}$, the proliferation of MCF- 

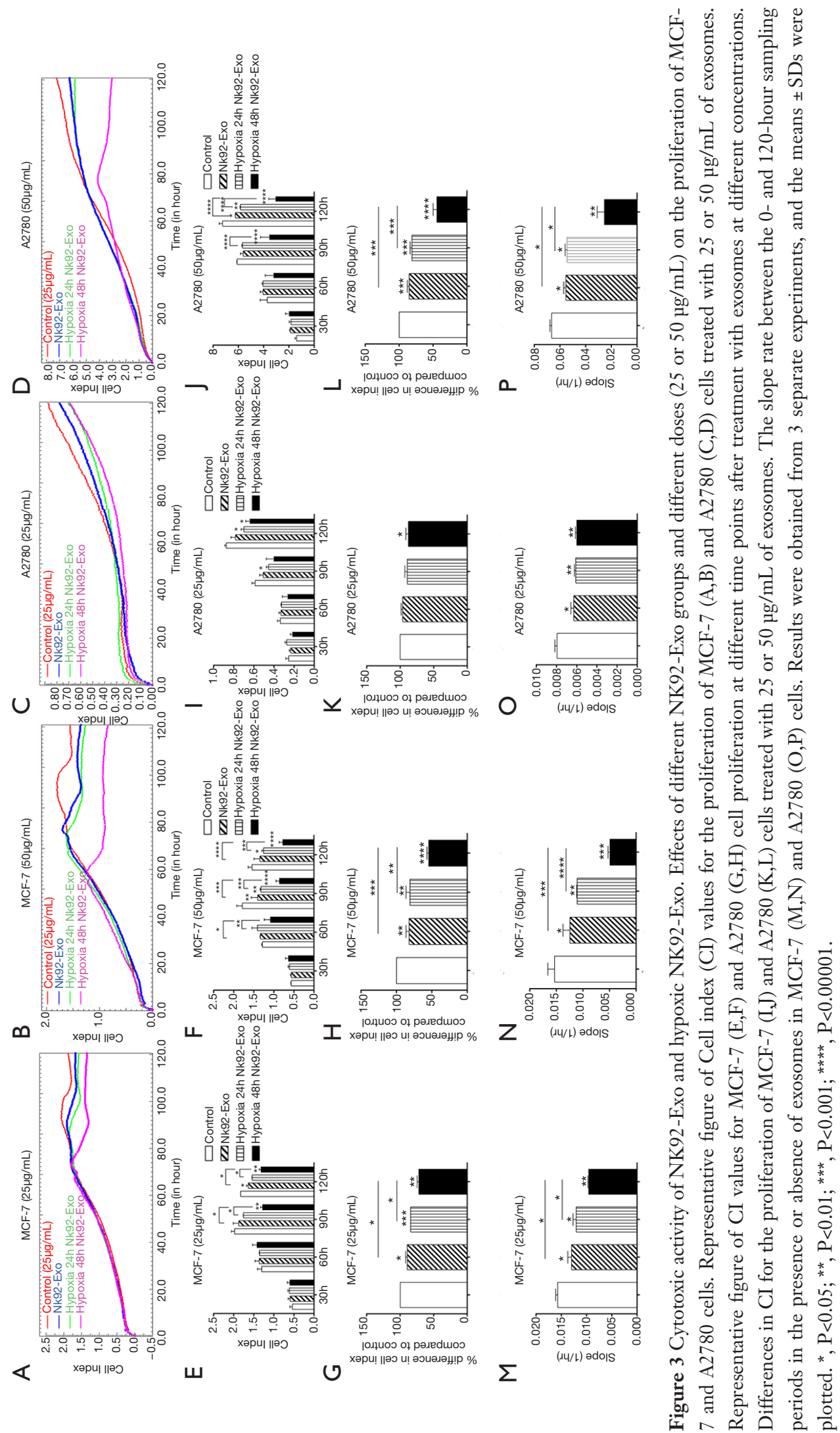
7 cells $(\mathrm{P}<0.05$; Figure $3 E, F, G, H)$ and $\mathrm{A} 2780$ cells $(\mathrm{P}<0.05$; Figure $3 I, \mathcal{F}, K, L)$ was inhibited compared to the control group. In particular, when the exosomal concentration was $50 \mu \mathrm{g} / \mathrm{mL}$ and the treatment duration was $90-120$ hours, the $\mathrm{CI}$ value of the target cells was significantly lower than that of the control group $(\mathrm{P}<0.05$; Figure $3 F, H, \mathcal{F}, L)$. However, from the CI value at 120 hours and the slope of the curve between 0 and 120 hours, it can be concluded that NK92Exo and 24-hour hypoxic NK92-Exo had similar inhibitory effects on target cells $(\mathrm{P}<0.05$; Figure $3 M, N, O, P)$. Results from this slope analysis indicated that different groups of exosomes altered the proliferation of target cells, with maximal effects being observed in the 48-hour hypoxic NK92-Exo group $(\mathrm{P}<0.01)$.

\section{Detection of cell migration ability via wound healing assay}

Results of the wound healing assay corroborated those of RTCA (Figure 4). We found that the migration rate of MCF-7 and A2780 cells declined in the NK92-Exo, 24-hour hypoxic NK92-Exo, and 48-hour hypoxic NK92-Exo groups compared with that in the respective MCF-7 $(\mathrm{P}<0.05$, $\mathrm{P}<0.01$; Figure $4 A, B)$ and $\mathrm{A} 2780(\mathrm{P}<0.001$; Figure $4 C, D)$ control groups. While these differences in decreasing migration were statistically significant between the 48-hour hypoxic NK92-Exo and NK92-Exo groups $(\mathrm{P}<0.05, \mathrm{P}<0.01$; Figure $4 B, D)$, they did not reach statistical significance between the 24-hour hypoxic NK92-Exo and NK92-Exo groups in the MCF-7 cells $(\mathrm{P}=0.98)$ or the A2780 cells $(\mathrm{P}=0.26)$ (Figure $4 B, D)$.

\section{Effects of NK-92 Exo on apoptosis of breast and ovarian cancer cells}

To evaluate the apoptosis induced by NK92-Exo, 24-hour hypoxic NK92-Exo, and 48-hour hypoxic NK92-Exo, pretreated MCF-7 and A2780 cells were stained with Annexin V-APC. The proportions of apoptotic MCF-7 cells after treatment with NK92-Exo, 24-hour hypoxic NK92Exo, and 48-hour hypoxic NK92-Exo were $68.19 \% \pm 4.19 \%$, $70.89 \% \pm 1.81 \%$, and $87.17 \% \pm 2.52 \%$, respectively $(\mathrm{P}<0.0$, $\mathrm{P}<0.01$, and $\mathrm{P}<0.01$, respectively; Figure $5 A, B)$, while the proportions of apoptotic A2780 cells were $21.20 \% \pm 1.22 \%$, $22.34 \% \pm 0.57 \%$, and $28.28 \% \pm 0.57 \%$, respectively $(\mathrm{P}<0.01$, $\mathrm{P}<0.001$, and $\mathrm{P}<0.001$, respectively; Figure 5C,D). Moreover, these results, as obtained by wound healing assay and flow cytometry (Figure $5 A, C$ ), were consistent with those of the RTCA, showing that treatment with NK92-Exo and 48-hour hypoxic NK92-Exo at the same concentrations reduced the proliferation of MCF-7 $(\mathrm{P}<0.05)$ and A2780 $(\mathrm{P}<0.05)$ cells. Our results revealed that FasL, perforin, and granzyme B were present in hypoxic NK92-Exo (Figure 5E); moreover, the levels of these proteins were significantly increased in 48-hour hypoxic NK92-Exo $(\mathrm{P}<0.05, \mathrm{P}<0.01$, and $\mathrm{P}<0.05$, respectively; Figure $5 F$ ).

\section{Cytotoxicity of hypoxic NK92-bIL15-Exo}

According to the previous results, we collected supernatant and performed exosome extraction after 48 hours of hypoxic treatment of NK92-hIL15 cells. The cytotoxicity of NK92-Exo and NK92-hIL15-Exo was measured by RTCA. Exosomes derived from NK92-hIL15 cells could obviously enhance the therapeutic effect in both MCF-7 and $\mathrm{A} 2780$ cells (Figure $6 A, B, \mathrm{P}<0.05, \mathrm{P}<0.01$ ). Moreover, hypoxic NK92-Exo and hypoxic NK92-hIL15-Exo showed higher cytotoxicity against cancer cells than did normoxic exosomes. In particular, hypoxic NK92-hIL15-Exo showed the strongest cytotoxicity among all groups of exosomes $(\mathrm{P}<0.05)$. Next, to identify the mechanism underlying the enhanced killing ability of hypoxic NK92-hIL15-Exo, an apoptosis assay was performed by using western blot to detect the expression of FasL, perforin, and granzyme B. Previous studies have shown that the expression of FasL and perforin is increased in NK-EV after IL-15 priming (27). In our study, the results indicated that exosomes from hIL-15modified NK cells under hypoxic conditions resulted in the enhancement of FasL, perforin, and granzyme B expression in NK cells $(\mathrm{P}<0.05, \mathrm{P}<0.01$, and $\mathrm{P}<0.001$, respectively; Figure $6 C, D)$.

\section{Discussion}

Due to their ability to reach tumor sites when loaded with drugs, microRNAs, or siRNAs, the application of extracellular vesicles as alternatives to liposomes in the tumor microenvironment has drawn considerable attention recently (28). Exosomes derived from NK92-MI cells have been found to inhibit hepatic stellate cell activation and liver fibrosis (29), and NK cell-derived exosomes entrapped with paclitaxel can enhance the antitumor effect (30). Accordingly, exosomes from NK cells may become a potential tool to treat cancer because of their cytotoxic activity against tumor targets (15). Here, we showed that exosomes derived from NK cells demonstrated a certain degree of cytotoxicity directed toward breast and ovarian 
A

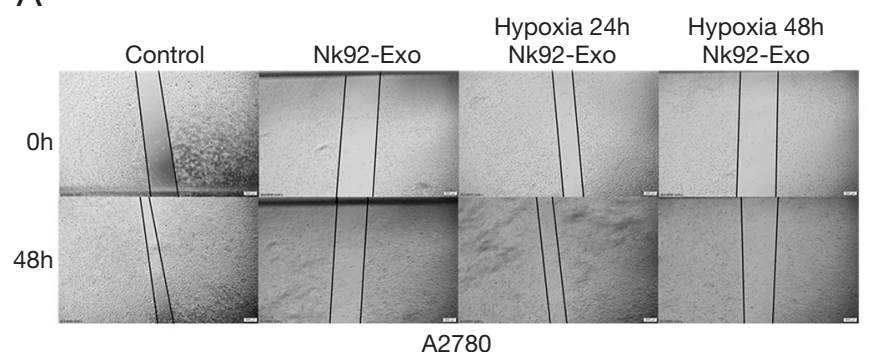

$\mathrm{A} 2780$

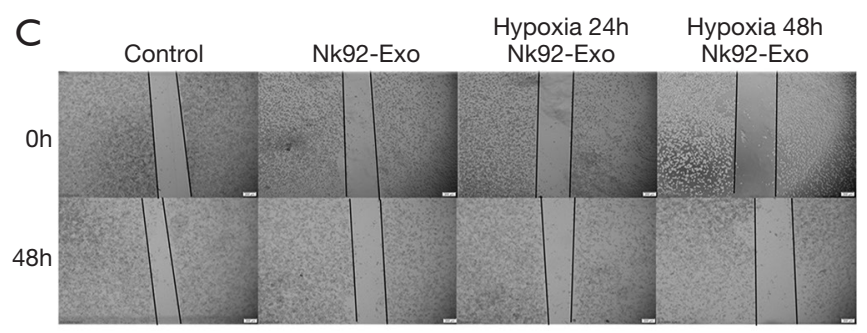

B

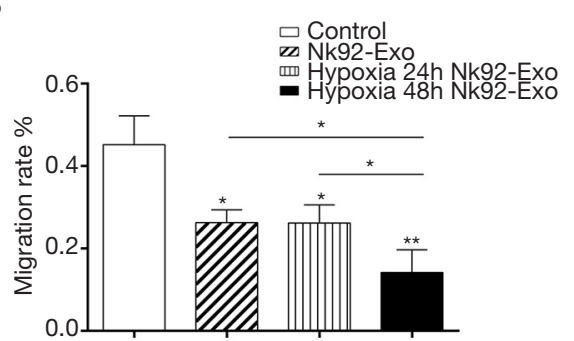

D

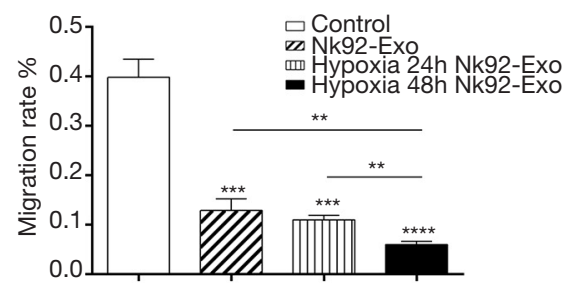

Figure 4 Detection of cell migration ability via wound healing assay. MCF-7 breast and A2780 ovarian cancer cells were co-cultured with different groups of NK92-Exo $(50 \mu \mathrm{g} / \mathrm{mL})$, with photos taken at the same observation point and at 0 and 48 hours post treatment (scale bar: $200 \mu \mathrm{m} ; \mathrm{A}, \mathrm{C})$. The scratch width was measured and cell mobility was calculated (B,D). Mobility = (initial scratch width - current scratch width)/initial scratch width $/ 2 \times 100 \%$. Results were obtained from 3 separate experiments, and the means \pm SDs were plotted. *, $\mathrm{P}<0.05 ;{ }^{* *}$, $\mathrm{P}<0.01 ;{ }^{* * *}, \mathrm{P}<0.001 ;{ }^{* * * *}, \mathrm{P}<0.00001$.

cancer cells, thus expanding their potential use in cancer treatment.

Hypoxia-induced exosomes aggravate tumor progression by delivering substances that promote tumor cell invasion and metastasis. According to Patton et al.'s report, increased exosome secretion was detected in human tubular epithelial cells under hypoxic conditions (21). Research has shown that exosome secretion increases under hypoxic conditions, and enrichment of exosome appears to be HIF-1 dependent $(31,32)$. Furthermore, the adaptive responses of breast and ovarian cancer cells to hypoxia also affect the tumor microenvironment, ultimately leading to invasive disease progression and treatment resistance. On the basis of previous research, we identified some of the detailed characteristics of exosomes derived from NK cells, and investigated the factors and hypoxic conditions that enhance the release of exosomes and the destructive effects of NK cells against tumor cells. The significance of this investigation is that the effects of hypoxia on the biogenesis of exosomes from NK cells remains unknown, nor is it clear as to whether these exosomes differ from NK exosomes.

In our study, we first treated NK92 cells under hypoxic conditions and detected the expression of HIF- $1 \alpha$, which increased with the prolongation of hypoxia duration (Figure 1).
We next observed that hypoxic stress stimulated the release of exosomes by NK92 cells, with the most consistent and noticeable effects observed in NK92 cells kept under hypoxic conditions for 48 hours. These findings are similar to those of a study that reported a hypoxia-induced increase in exosome secretion in the ureteral obstructed kidney (21). Characterization of hypoxic NK92-Exo showed that these exosomes were membrane structures of 50-200 nm in size. As demonstrated by western blot, hypoxic NK92-Exo obtained at 24 and 48 hours displayed CD63 and TSG101 expression (Figure $2 A, B, C, D$ ), which are 2 proteins that have been regarded as exosomal markers in previous studies (33). Within the hypoxia group, expression levels of CD63 and TSG101 were slightly increased (Figure 2D). Previous studies showed that ISGylation of MVB TSG101 protein blocked exosome secretion by inducing the aggregation and degradation of exosomes (34). We found that TSG101 expression in exosomes increased with the prolongation of hypoxic treatment of NK cells. Although the numbers of NK92 cells cultured under normoxic and hypoxic conditions were the same, the yield of exosomes obtained from NK92 cells under hypoxic treatment was increased compared to that obtained from NK92 cells cultured under normoxic conditions (Figure 2E). Thus, our results showed that while 
A

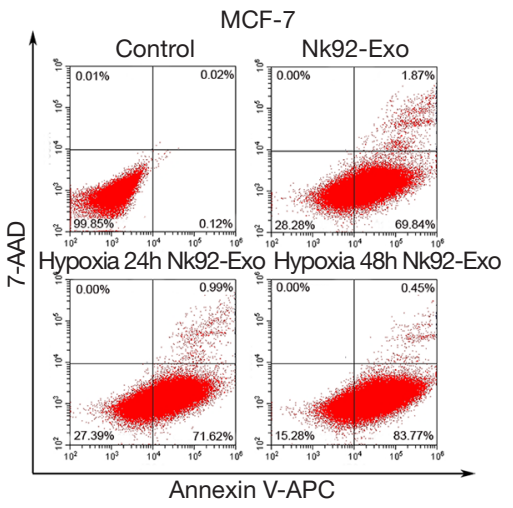

C

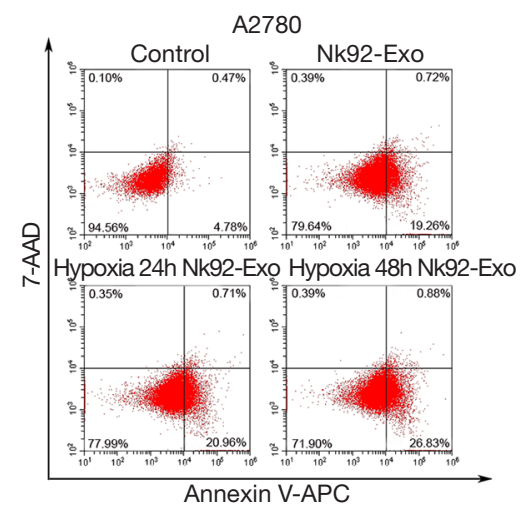

$\mathrm{E}$

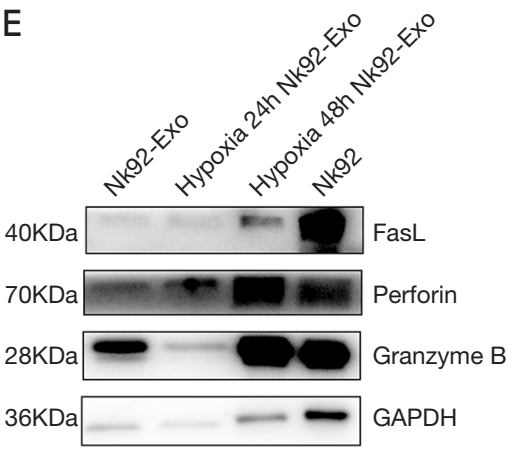

B

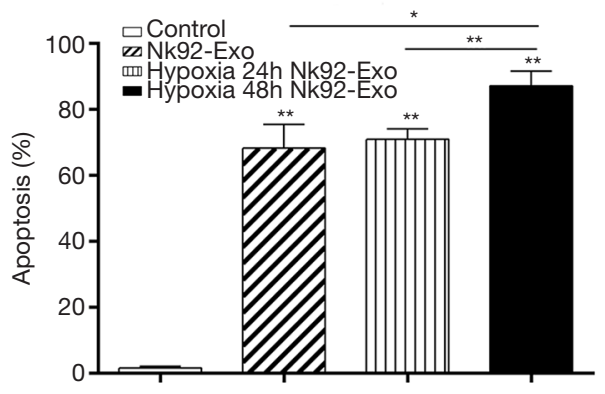

D

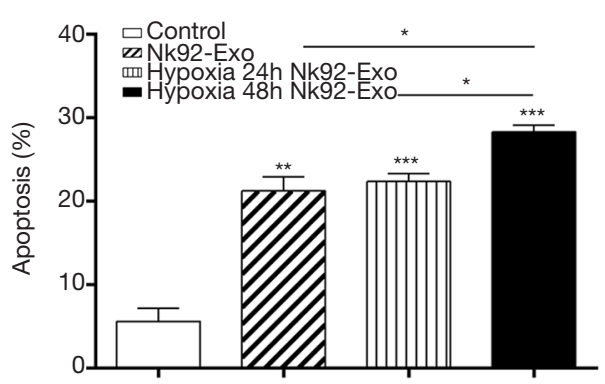

$\mathrm{F}$

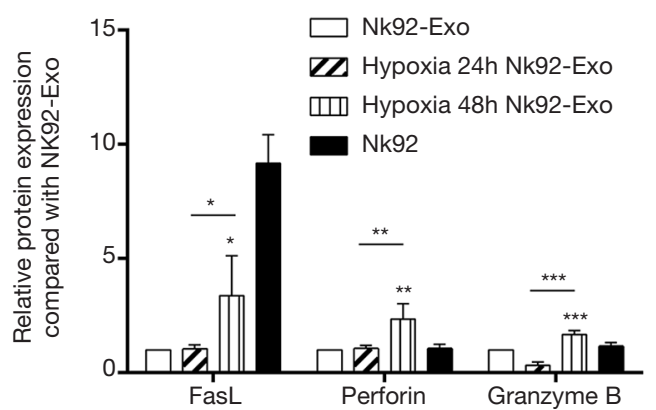

Figure 5 Hypoxia NK92-Exo induced apoptosis of breast and ovarian cancer cells. Detection of apoptosis in MCF-7 and A2780 cells subjected to different treatments of NK92-Exo and assessed by flow cytometry (FCM) (A,C). Statistical analysis of apoptosis (B,D). Apoptotic rates of MCF-7 and A2780 cells were significantly increased by treatment with 48-hour hypoxic NK92-Exo. Total proteins (30 $\mathrm{gg})$ from NK92-Exo, 24-hour hypoxic NK92-Exo, and 48-hour hypoxic NK92-Exo were loaded into each lane. The proteins FasL, perforin, and granzyme B were expressed in both NK cells and exosomes (E). The levels of FasL, perforin, and granzyme B in NK92-Exo, 24-hour hypoxic NK92-Exo, and 48-hour hypoxic NK92-Exo were estimated using the Image J software (F). The 48-hour hypoxic NK92Exo lysates contained higher levels of FasL, perforin, and granzyme B than the NK92-Exo lysates. Results were obtained from 3 separate experiments, and the means \pm SDs were plotted. *, $\mathrm{P}<0.05 ;{ }^{* *}, \mathrm{P}<0.01 ;{ }^{* * *}, \mathrm{P}<0.001$.

hypoxia did not significantly alter the morphology or size of NK92-Exo, it did increase exosome production.

Breast cancer and ovarian malignant tumors are the most common malignant tumors in women. Symptoms of ovarian and breast cancer are quite subtle in the early stages; therefore, the disease is usually at an advanced stage at the time of diagnosis and represents a serious threat (35). Here, we have shown that exosomes from NK cells under 


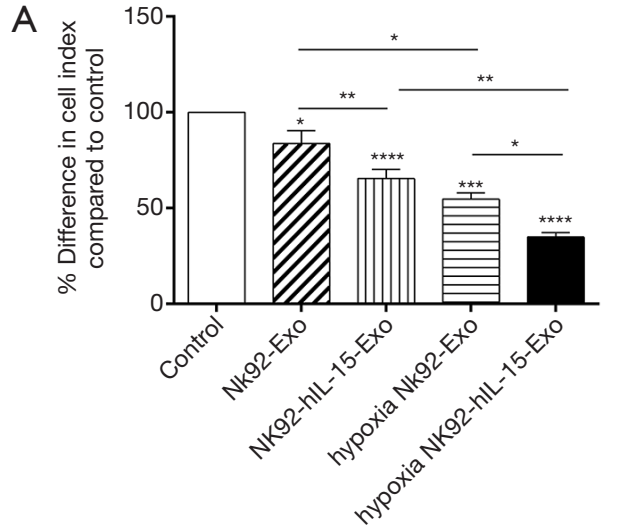

C

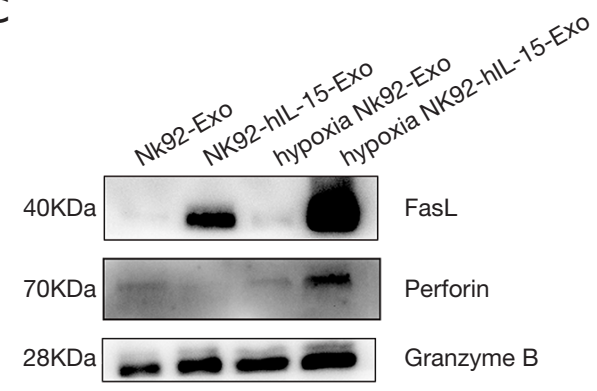

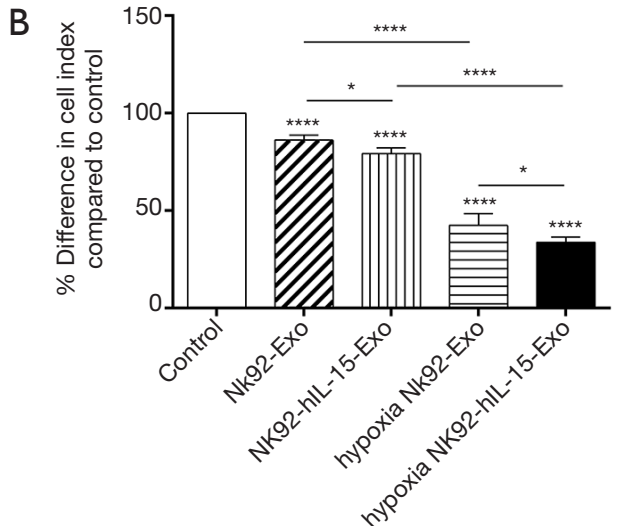

D

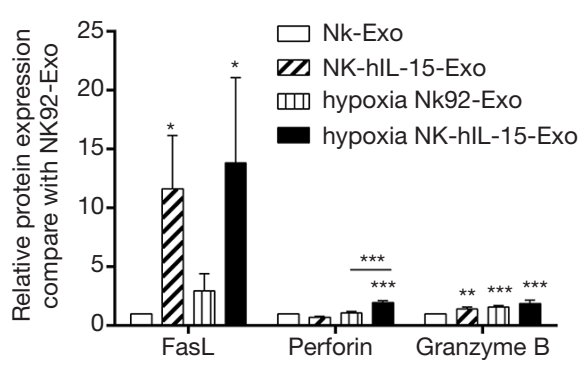

Figure 6 Cytotoxicity of NK92-hIL15-Exo under hypoxic conditions. Effects of the NK92-Exo and NK92-hIL15-Exo under normoxic or hypoxic conditions on the viability of MCF-7 and A2780 cells were measured by RTCA. Differences in the cell index compared to the control group were calculated for different groups of exosomes $(50 \mu \mathrm{g} / \mathrm{mL})(\mathrm{A}, \mathrm{B})$. NK92-Exo and NK92-hIL15-Exo were analyzed for FasL, perforin, and granzyme B expression by western blotting $(\mathrm{C}, \mathrm{D})$. Results were obtained from 3 separate experiments, and the means $\pm \mathrm{SDs}$ were plotted. *, $\mathrm{P}<0.05$; **, $\mathrm{P}<0.01$; *** $\mathrm{P}<0.001$; ${ }^{* * *}, \mathrm{P}<0.0001$.

hypoxic conditions display antitumor effects against MCF7 breast and A2780 ovarian cancer cells, as demonstrated by RTCA and wound healing assays (Figures 3,4). Results from previous studies have shown that exosomes derived from NK92 cells have significant antitumor effects on both melanomas and lymphomas $(15,28)$. Similarly, our current results show that exosomes obtained from NK92 cells inhibited the proliferation and migration of MCF-7 and A2780 cells, and these effects were enhanced under hypoxic conditions, as revealed by RTCA and wound healing assays. However, the A2780 cells were not particularly sensitive to NK92 cell-derived exosomes, with the exosomes showing a strong cytotoxic effect at a concentration of $50 \mu \mathrm{g} / \mathrm{mL}$, and not at $25 \mu \mathrm{g} / \mathrm{mL}$. Furthermore, we found that the cytotoxic effects of exosomes significantly increased with the duration of hypoxic treatment, with maximal effects being observed in response to 48-hour exposure to hypoxic conditions.

Apoptosis is one of the important mechanisms of NK cell immunotherapy $(30,36)$. This result is consistent with our flow cytometry results, which showed that hypoxia promoted the early and late apoptosis of target cells (Figure $5 A, C$ ). In particular, after 48 hours of hypoxia, the ability of NK92 cells to release exosomes that induced target cell apoptosis was more than double that of the normoxic group.

Perforin is a core molecule required by NK cells to induce apoptosis and participate in tumor destruction mediated by death receptor pathways, including FasL and tumor necrosis factor-related apoptotic ligand (TRAIL) (37). Studies have shown that exosomes from NK cells contain both FasL and perforin (15). In this study, our western blot results demonstrated that hypoxic NK92-Exo contain FasL, perforin, and granzyme B proteins (Figure $5 E, F$ ), and the levels of these proteins were increased in the 48-hour hypoxia NK92-Exo group compared with the NK92Exo group (Figure $5 E$ ). In this way, exosomes secreted by NK92 cells treated with hypoxia for 48 hours were more 
cytotoxic and contained greater levels of the toxic proteins FasL, perforin, and granzyme B as compared with the other groups generating exosomes at identical concentrations.

Studies have shown that the use of exogenous hIL-15 can raise the yield of exosomes from NK cells and enhance the cytotoxic effect. In our study, we found that exosomes from the cells treated under hypoxic conditions could obtain similar results and also enhance the cytotoxicity. Therefore, to obtain more cytotoxic exosomes, we first transduced the cytokine hIL-15 into NK92 cells and obtained exosomes after hypoxic treatment. We found that exosomes obtained under these conditions showed increased cytotoxicity (Figure 6).

The complexity of the tumor microenvironment affects the efficacy of immune cells in the treatment of cancer, which can reduce the effectiveness of NK cell immunotherapy. For instance, hypoxia surrounding the tumors has been reported to reduce the secretion of perforin and granzymes A and B, inhibit Fas/FasL interactions, and decrease the expression of natural cytotoxicity receptors (38-40). However, we believe that hypoxia activates NK cells and releases exosomes to play a cytotoxic and pro-apoptotic role. However, lowoxygen environments favor the accumulation and delivery of exosomes, as low oxygen values can attract exosomes and promote membrane fusion (21). Moreover, hypoxia favors the accumulation and delivery of exosomes, as a low oxygen concentration can have the effect of attracting these exosomes and promoting their membrane fusion. Although NK92 cells have been well established for adoptive immunotherapy, they are not as safe for use as exosomes from NK92 cells. The reduced safety of NK92 cells is related to their derivation from non-Hodgkin lymphoma patients. Therefore, hypoxia can promote the production of NK92-Exo and hypoxic NK92-hIL15-Exo and would be more effective when used in immunotherapy.

In conclusion, we found that exosomes derived from NK92 or NK92-hIL15 cells under hypoxic conditions are more potent than those from NK92 cells in inhibiting the proliferation and migration of, while promoting apoptosis of cancer cells.

\section{Acknowledgments}

Funding: This work was supported by grants from the National Natural Science Foundation of China (81370730 and 81571512), the Science and Technology Plan of Yantai City Government (2019MSGY134), the Key Program of the Natural Science Foundation of Shandong Province (ZR2015JL027), Clinical Medicine Discipline Construction
Project (20037720), Shandong Province Medical and Health Science and Technology Development Plan (2017WS361) and Yantai Double Hundred plan.

\section{Footnote}

Reporting Checklist: The authors have completed the MDAR reporting checklist. Available at http://dx.doi.org/10.21037/ atm-21-347

Data Sharing Statement: Available at http://dx.doi. org/10.21037/atm-21-347

Conflicts of Interest: All authors have completed the ICMJE uniform disclosure form (available at http://dx.doi. org/10.21037/atm-21-347). The authors have no conflicts of interest to declare.

Ethical Statement: The authors are accountable for all aspects of the work in ensuring that questions related to the accuracy or integrity of any part of the work are appropriately investigated and resolved.

Open Access Statement: This is an Open Access article distributed in accordance with the Creative Commons Attribution-NonCommercial-NoDerivs 4.0 International License (CC BY-NC-ND 4.0), which permits the noncommercial replication and distribution of the article with the strict proviso that no changes or edits are made and the original work is properly cited (including links to both the formal publication through the relevant DOI and the license). See: https://creativecommons.org/licenses/by-nc-nd/4.0/.

\section{References}

1. Wu Y, Li J, Jabbarzadeh Kaboli P, et al. Natural killer cells as a double-edged sword in cancer immunotherapy: A comprehensive review from cytokine therapy to adoptive cell immunotherapy. Pharmacol Res 2020;155:104691.

2. Cooper MA, Fehniger TA, Caligiuri MA. The biology of human natural killer-cell subsets. Trends Immunol 2001;22:633-40.

3. Freud AG, Mundy-Bosse BL, Yu J, et al. The Broad Spectrum of Human Natural Killer Cell Diversity. Immunity 2017;47:820-33.

4. Dogra P, Rancan C, Ma W, et al. Tissue Determinants of Human NK Cell Development, Function, and Residence. Cell 2020;180:749-763.e13. 
5. Michel T, Poli A, Cuapio A, et al. Human CD56bright NK Cells: An Update. J Immunol 2016;196:2923-31.

6. Dou R, Hong Z, Tan X, et al. Fas/FasL interaction mediates imbalanced cytokine/cytotoxicity responses of iNKT cells against Jurkat cells. Mol Immunol 2018;99:145-53.

7. Prager I, Watzl C. Mechanisms of natural killer cell-mediated cellular cytotoxicity. J Leukoc Biol 2019;105:1319-29.

8. McNerney KO, Karageorgos SA, Hogarty MD, et al. Enhancing Neuroblastoma Immunotherapies by Engaging iNKT and NK Cells. Front Immunol 2020;11:873.

9. Minetto P, Guolo F, Pesce S, et al. Harnessing NK Cells for Cancer Treatment. Front Immunol 2019;10:2836.

10. Tonn T, Schwabe D, Klingemann HG, et al. Treatment of patients with advanced cancer with the natural killer cell line NK-92. Cytotherapy 2013;15:1563-70.

11. Gurunathan S, Kang MH, Jeyaraj M, et al. Review of the Isolation, Characterization, Biological Function, and Multifarious Therapeutic Approaches of Exosomes. Cells 2019;8:307.

12. Yáñez-Mó M, Siljander PR, Andreu Z, et al. Biological properties of extracellular vesicles and their physiological functions. J Extracell Vesicles 2015;4:27066.

13. Jadli AS, Ballasy N, Edalat P, et al. Inside(sight) of tiny communicator: exosome biogenesis, secretion, and uptake. Mol Cell Biochem 2020;467:77-94.

14. Lugini L, Cecchetti S, Huber V, et al. Immune surveillance properties of human NK cell-derived exosomes. J Immunol 2012;189:2833-42.

15. Zhu L, Kalimuthu S, Gangadaran P, et al. Exosomes Derived From Natural Killer Cells Exert Therapeutic Effect in Melanoma. Theranostics 2017;7:2732-45.

16. Zhang B, Chen Y, Shi X, et al. Regulation of branchedchain amino acid metabolism by hypoxia-inducible factor in glioblastoma. Cell Mol Life Sci 2021;78:195-206.

17. Cui C, Fu K, Yang L, et al. Hypoxia-inducible gene 2 promotes the immune escape of hepatocellular carcinoma from nature killer cells through the interleukin-10-STAT3 signaling pathway. J Exp Clin Cancer Res 2019;38:229.

18. Vaupel P, Multhoff G. Hypoxia-/HIF-1 $\alpha$-Driven Factors of the Tumor Microenvironment Impeding Antitumor Immune Responses and Promoting Malignant Progression. Adv Exp Med Biol 2018;1072:171-5.

19. Su Q, Wang J, Fan M, et al. Sanguinarine disrupts the colocalization and interaction of HIF- $1 \alpha$ with tyrosine and serine phosphorylated-STAT3 in breast cancer. J Cell Mol Med 2020;24:3756-61.
20. Mittal K, Aneja R. Spotlighting the hypoxia-centrosome amplification axis. Med Res Rev 2020;40:1508-13.

21. Patton MC, Zubair H, Khan MA, et al. Hypoxia alters the release and size distribution of extracellular vesicles in pancreatic cancer cells to support their adaptive survival. J Cell Biochem 2020;121:828-39.

22. Ou ZL, Luo Z, Wei W, et al. Hypoxia-induced shedding of MICA and HIF1A-mediated immune escape of pancreatic cancer cells from NK cells: role of circ_0000977/miR-153 axis. RNA Biol 2019;16:1592-603.

23. Multhoff G, Vaupel P. Hypoxia Compromises Anti-Cancer Immune Responses. Adv Exp Med Biol 2020;1232:131-43.

24. Gong JH, Maki G, Klingemann HG. Characterization of a human cell line (NK-92) with phenotypical and functional characteristics of activated natural killer cells. Leukemia 1994;8:652-8

25. Théry C, Amigorena S, Raposo G, et al. Isolation and characterization of exosomes from cell culture supernatants and biological fluids. Curr Protoc Cell Biol 2006; Chapter 3:Unit 3.22.

26. Dowling CM, Herranz Ors C, Kiely PA. Using realtime impedance-based assays to monitor the effects of fibroblast-derived media on the adhesion, proliferation, migration and invasion of colon cancer cells. Biosci Rep 2014;34:e00126.

27. Zhu L, Kalimuthu S, Oh JM, et al. Enhancement of antitumor potency of extracellular vesicles derived from natural killer cells by IL-15 priming. Biomaterials 2019;190-191:38-50.

28. Di Pace AL, Tumino N, Besi F, et al. Characterization of Human NK Cell-Derived Exosomes: Role of DNAM1 Receptor In Exosome-Mediated Cytotoxicity Against Tumor. Cancers (Basel) 2020;12:661.

29. Wang L, Wang Y, Quan J. Exosomes derived from natural killer cells inhibit hepatic stellate cell activation and liver fibrosis. Hum Cell 2020;33:582-9.

30. Han D, Wang K, Zhang T, et al. Natural killer cell-derived exosome-entrapped paclitaxel can enhance its anti-tumor effect. Eur Rev Med Pharmacol Sci 2020;24:5703-13.

31. Zhang G, Zhang Y, Cheng S, et al. CD133 positive U87 glioblastoma cells-derived exosomal microRNAs in hypoxia- versus normoxia-microenviroment. J Neurooncol 2017;135:37-46.

32. Kumar A, Deep G. Exosomes in hypoxia-induced remodeling of the tumor microenvironment. Cancer Lett 2020;488:1-8.

33. Kalluri R, LeBleu VS. The biology, function, and biomedical applications of exosomes. Science 
2020;367:eaau6977.

34. Villarroya-Beltri C, Baixauli F, Mittelbrunn M, et al. ISGylation controls exosome secretion by promoting lysosomal degradation of MVB proteins. Nat Commun 2016;7:13588.

35. Maennling AE, Tur MK, Niebert M, et al. Molecular Targeting Therapy against EGFR Family in Breast Cancer: Progress and Future Potentials. Cancers (Basel) 2019;11:1826.

36. Wu CH, Li J, Li L, et al. Extracellular vesicles derived from natural killer cells use multiple cytotoxic proteins and killing mechanisms to target cancer cells. J Extracell Vesicles 2019;8:1588538.

37. Sordo-Bahamonde C, Lorenzo-Herrero S, Payer ÁR, et al. Mechanisms of Apoptosis Resistance to NK Cell-Mediated
Cytotoxicity in Cancer. Int J Mol Sci 2020;21:3726.

38. Baginska J, Viry E, Paggetti J, et al. The critical role of the tumor microenvironment in shaping natural killer cell-mediated anti-tumor immunity. Front Immunol 2013;4:490.

39. Ames E, Hallett WH, Murphy WJ. Sensitization of human breast cancer cells to natural killer cell-mediated cytotoxicity by proteasome inhibition. Clin Exp Immunol 2009;155:504-13.

40. Balsamo M, Manzini C, Pietra G, et al. Hypoxia downregulates the expression of activating receptors involved in NK-cell-mediated target cell killing without affecting ADCC. Eur J Immunol 2013;43:2756-64.

(English Language Editor: J. Reynolds)

Cite this article as: Jiang Y, Jiang H, Wang K, Liu C, Man X, $\mathrm{Fu} \mathrm{Q}$. Hypoxia enhances the production and antitumor effect of exosomes derived from natural killer cells. Ann Transl Med 2021;9(6):473. doi: 10.21037/atm-21-347 